\title{
Bronchoalveolar lavage: perspective from the 11th BAL Conference
}

\author{
H.Y. Reynolds
}

Monaldi Arch Chest Dis 2008; 69: 3, 91-93.

Medical Officer, Division on Lung Diseases, National Heart, Lung, and Blood Institute, Bethesda, MD, U.S.A.

Corespondence: Herbert Y. Reynolds, M.D., Medical Officer, Division on Lung Diseases, National Heart, Lung, and Blood Institute, 6701 Rockledge Drive \# 10180, Bethesda, MD 20892, U.S.A.; e-mail: Reynoldh@nhlbi.nih.gov

\section{Introduction}

Bronchial lavage of the human airways, as therapy to remove secretions, dates to the early use of the rigid bronchoscope over a century ago (1). And for the past 50 years, lung biological specimens have been obtained also for research by washing airways through rubber tubes anchored in the bronchi (1). Limited lavage in normal subjects to recover airway samples for research began in 1967 (2). Availability of the flexible bronchofiberscope (3) in the late 1960's popularized the use of bronchoscopy, and the recovery of lung washings for clinical analysis and research studies was done frequently. We studied airway cells and protein and immune components in lavage fluid from normal subjects to describe the lower respiratory tract milieu in 1974 (4). Subsequently, the use of bronchoalveolar lavage (BAL) to retrieve specimens from patients with many forms of lung disease and from controls or normals spread quickly throughout the US, Europe and Japan (5). There was sufficient interest in presenting and discussing lung research prompted by BAL that the first BAL Conference was held in 1979 (6). Dr. Ronald Crystal, Dr. Anthony Kalica and I organized the second BAL Conference in Columbia Maryland in 1984 (7). Nine others have followed including the 11 th BAL Conference in Athens, Greece on June 19-21, 2008, which is the subject of this report.

\section{1 th BAL International Conference}

This was the first joint BAL and WASOG meeting, and this was a plausible plan as there was considerable cross over between the research findings that used BAL sampling to obtain biological specimens from the lung and others presented that analyzed and interpreted lung-derived biomarkers found in many interstitial lung diseases (ILD). Thus, the respective BAL and WASOG presentations interdigitated well. Among the formal presentations at plenary sessions, 18 were related to BAL sampling. Among almost 100 posters, 25 were re- search studies involved with BAL. The posters were all presented by their authors during several organized "poster rounds"; three were selected for oral poster presentations in plenary sessions. This analysis will emphasize the oral BAL studies.

In organizing a summary of noteworthy contributions from BAL studies, these have been grouped into 6 categories:

\section{Analysis of BAL Findings to Evaluate Disease(s)}

The study of cells and proteinacious substances in lung washings continues to provide insight into the pathogenesis and host immunity responses involved in inflammation, fibrosis, acute injury, asthma, and infection. Because components retrieved in BAL fluid are in close proximity to diseased tissue and are the first approximation to in situ occurring events, relevant new observations were given.

Dr. V. Potelli (Italy), discussed BAL findings in acute lung injury that might be a surrogate for a lung tissue biopsy. Dr. D. Israel-Biet (France), evaluating patients after lung transplantation for graft rejection and onset of the bronchiolitis obliterans syndrome, looked for markers of these conditions; persistence of polymorphonuclear neutrophils appeared to be a signal. As environmental exposure is a common cause or confounding factor in establishing a precise etiology of ILD, the BAL findings were presented for several common diseases. Dr. S. Constantopoulis (Greece) assessed the role of BAL in non-occupational asbestos exposure, Dr. F. Kokknis presented about chrysotile exposure, and D. F. Evyapan did so for metal induced lung disease. Dr. M. Drent (Netherlands) updated the usefulness of a computer data bank of BAL components available for analysis in establishing a diagnosis of a particular ILD.

\section{Technology Applied to BAL Continues to Evolve}

New analytical modalities continue to reveal more biomarkers and mechanisms defining cellu- 
lar activities. With the expanding "omics" approach to biologic materials a much more comprehensive analysis of cellular output and gene displays in BAL fluid and cells can be viewed from a normal or patient specimen. Dr. P. Rottoli, (Italy), a pioneer in proteomics, continues to use this tool to search for biomarkers in ILD. Similarly, gene arrays from cells, as described by Dr. N. Kamins$\mathrm{ki}$, (USA), sampled from lung tissue, BAL, and blood described cellular activity in ILD and for many other diseases.

\section{Timing of BAL Fluid Samples May Reveal an Evolution of Changes}

As BAL is well tolerated by normals (controls) and patients, the samplings can be repeated. This may have value in monitoring changes in BAL components during early lung development and subsequent aging, and during persistent illness. Dr. D. Phelps (USA), described age-related changes in BAL components with a rat model. Similarly, Dr. N. Kaminski (USA) discussed serial analyses on blood cells, but which could apply to BAL cells. Longitudinal evaluation to monitor airway BAL changes is to be encouraged in patients with evolving or persistent illness, and in volunteer controls to observe changes that occur normally with aging. Providing BAL data from older control subjects would better approximate or contrast with these found in older patients. Controls for research often need to be better age-approximated with patients, particularly in metabolomic comparisons and in drug turnover rates when pharmacomics are investigated.

\section{Special Cells Isolated from BAL}

Lung lavage retrieves important detachable types of cells and other biological specimens for in vitro study, especially alveolar macrophages (8) and lymphocytes. Thus, a method to isolate dendritic cells (DC's) from BAL fluids was of particular note (9). Dr. T. Berge and colleagues (Netherlands) and Dr. B. Lambrecht (Belgium) presented a method to obtain dendritic cells; they estimate that about $0.1 \%$ of normal lavage cells are DC's. The ability to recover DC's among alveolar cells, which are mostly macrophages, provides a means for dissecting the innate immunity processing that begins in the alveolar space. The in vitro study of alveolar macrophages as phagocytes and effector cells in initiating inflammation and alveolitis has been considerable (8). But, Dr. C. Saltini's (Italy) use of them to reproduce the kinetics of tuberculosis infection, seemingly replicates an "in vivo" approach to creating a Mycobacterium exposure to AM's with subsequent infection of $40 \%$ of these cells and then elucidating their production of inflammatory cytokines. This seems the way tuberculosis may begin in a susceptible person.

\section{Perturbations in Animals or Humans that Affect BAL Components}

Inhalation of cigarette smoke, exposure to various environmental toxins, often occupation-relat- ed, and aspiration of refluxed gastric secretions, all induce special changes in the composition of BAL components. Another exposure illustration with ozone by Dr. J. Floros (USA) created oxidative stress in the airways that was found to effect surfactant protein A. The result was illustrated with a proteomics analysis that introduced the term "discovery proteomics".

\section{Use of BAL in Children}

As this procedure is finding more applications in young patients, now performed shortly after birth in some with congenital diseases such as cystic fibrosis, and in children with asthma or interstitial lung diseases, the safety and consequences of the lavage procedure require continued surveillance.

It was in part this consideration of safety with research use of investigative fiberoptic bronchoscopy, often coupled with broncho-provocation to simulate asthma and then performing lavage, and/or other procedures done such as airway brushing and transmucosal or transbronchial biopsies that prompted the National Heart, Lung, and Blood Institute and the National Institute of Allergy and Infectious Diseases to convene a workshop to review the scientific merit gained from this approach (10). An Appendix is available online in the journal issue (www.atsjournals.org) that was included with the Workshop Summary (titled: Appendix II: "Ethical Issues Related to Bronchoprovocation and Bronchoscopy Research"). The consensus among the workshop participants was positive that the risks compared with new scientific information obtained was worthwhile; continued use of this form of research was reaffirmed. But, there were some concerns about adequate training of personnel performing these procedures, and obtaining surrogate permission for these young, vulnerable patient subjects that should be reviewed for possible updating. Other issues considered included: informed consent, financial incentives, and additional protections for research involving children and those subjects who need surrogate permissions.

\section{What Might Be Future Expectations from BAL Analysis?}

In several years, a next BAL Conference will occur in Maastricht, The Netherlands, on June 1518, 2011. What should we expect this sampling procedure to yield?

\section{Continued Revelations From Research on BAL Samples Will Continue}

As this method of obtaining airway-alveolar space biological specimens already has contributed many important insights into the normal and diseased respiratory tract, more scientific observations will be forthcoming. Although this sampling method is termed "BAL," operationally the procedure is broader and encompasses other strate- 
gies (11) which should be given more innovation, such as local sampling in large versus peripheral airways, analysis of exhaled breath condensates, comparisons between blood and airway cells and proteins, and genetic arrays of respiratory cells. As the "omics" wave of measurement technologies continues, the discovery of new substances or cellular functions will enhance the understanding of many diseases. Perhaps more research will occur that compares the upper respiratory tract secretions and cells with the lower tract that might find similarities that would promote more upper airway sampling. This could facilitate less invasive longitudinal monitoring, be acceptable for children and other younger subjects, and explore diseases that affect both portions of the respiratory tract, such as allergic rhinitis-sinusitis and hyperactive airways of asthma syndromes.

\section{Training of Future Respiratory Clinical Sci- entists Who Use Respiratory Tract Sampling Methods}

The continued output of relevant scientific research on respiratory tract diseases is predicated on having a sufficient supply of interested, well trained, innovative investigators. How we motivate and support this next generation is an important task for the academic research community (12, 13). The success to fund and train these investigators is a collective effort between the supporting agencies, the professional societies and specialty groups, and the pharmaceutical industry. An assessment of "how are we doing" might be considered (14). The BAL Conference with it's broadly representative and international composition of participants might consider a formal review of some of these training topics.

\section{BAL Will Help to Reveal "Secrets" Still in the Lungs}

As it remains surprising what "we don't know" about cells in the respiratory tract and their functions, a NHLBI workshop was held (July 9-10, 2007) to explore how more information can be obtained about still unrecognized and insufficiently studied cells. The summary of this workshop and companion papers that review in detail the development of the lung, the airways, the alveolar unit, and the pulmonary vasculature will each present research recommendations (15). The use of BAL and other local sampling methods to retrieve cells and other biological specimens will be needed. Fu- ture BAL conferences will likely deal with these new findings, as the lung's cellular secrets are revealed.

\section{References}

1. Reynolds HY. Bronchoalveolar Lavage- state of art. Amer Rev Respir Dis 1987; 135: 250-263.

2. Finley TN, Swenson EW, Curran WS, et al. Bronchopulmonary Lavage in normal subjects and patients with obstructive lung disease. Ann Intern Med 1967; 66: 651-658.

3. Ikeda S, Yanai N, Ishikawa S. Flexible Bronchofiberscope. Keio J Med 1968; 17: 1-16.

4. Reynolds HY, Newball HH. Analysis of Proteins and Respiratory Cells Obtained from Human Lungs by Bronchial Lavage. J Lab Clin Med 1974; 84: 559-573.

5. Reynolds HY. Use of Bronchoalveolar Lavage in $\mathrm{Hu}-$ mans-Past Necessity and Future Imperative. Lung 2000; 178: 271-293.

6. Le Lavage Broncho-Alveolaire chez l'homme-colloque: Proceedings edited by Biserte G, Chrétien J, Voisin C. Institut National de La Santé et de la Recherche Médicale, rue de Tolbiac, Paris, France; 1979: 1-544.

7. Crystal, RG, Reynolds HY, Kalica, AR. Bronchoavleolar Lavage. The report of an International Conference. Chest 1986; 90:122-131.

8. Reynolds HY. Lung Inflammation and Fibrosis: An Alveolar Macrophage-centered Perspective from the 1970s to 1980s. Am J Respir Crit Care Med 2005; 171: 98-102.

9. Reynolds HY. Advances in Understanding Pulmonary Host Defense Mechanisms: Dendritic Cell Function and Immunomodulation. Current Opinion in Pulm Med 2000; 6: 209-216.

10. Busse WW, Wanner A, Adams K, Reynolds HY, et al. NHLBI/NIAID Workshop. Investigative Bronchoprovocation and Bronchoscopy in Airway Diseaes. Am J Respir Crit Care Med 2005; 172: 807-816.

11. Reynolds HY. Sampling Local Respiratory Tract Sites for Inflammation. Sarcoidosis Vasc Diffuse Lung Dis 2001; 18: 138-148.

12. Reynolds HY. Choosing a Research Health Career, Mentoring is Essential. Lung 2008; 186: 1-6.

13. Reynolds HY. Mentoring - Nurturing Clinician and Physician Scientists in an Academic Career. Pharos 2007; 70: 26-28.

14. Reynolds HY, Rothgeb A, Colombini-Hatch S, Gail DB, Kiley JP. The Pipeline: Preparing and Training Pulmonary Scientists for Research Careers. Lung 2008; 186: 279-291.

15. Franks TJ, Colby TV, Travis WD, Tuder RM, Reynolds HY, and Workshop participants. NHLBI Workshop. Resident Cellular Components of the Human Lung: Current Knowledge and Goals for Research on Cell Phenotyping and Function. Proc Amer Thoracic Soc 2008; 5: 763-766. 